PROCEEDINGS OF THE

AMERICAN MATHEMATICAL SOCIETY

Volume 137, Number 4, April 2009, Pages 1431-1437

S 0002-9939(08)09651-2

Article electronically published on October 27, 2008

\title{
ANALYTICITY OF THE SRB MEASURE FOR HOLOMORPHIC FAMILIES OF QUADRATIC-LIKE COLLET-ECKMANN MAPS
}

\author{
VIVIANE BALADI AND DANIEL SMANIA
}

(Communicated by Jane M. Hawkins)

\begin{abstract}
We show that if $f_{t}$ is a holomorphic family of quadratic-like maps with all periodic orbits repelling so that for each real $t$ the map $f_{t}$ is a real Collet-Eckmann $S$-unimodal map, then, writing $\mu_{t}$ for the unique absolutely continuous invariant probability measure of $f_{t}$, the map$$
t \mapsto \int \psi d \mu_{t}
$$

is real analytic for any real analytic function $\psi$.
\end{abstract}

\section{IntRoduction AND STATEMENT OF THE THEOREM}

If $t \mapsto f_{t}$ is a smooth one-parameter family of dynamics $f_{t}$ so that $f_{0}$ admits a unique SRB measure $\mu_{0}$, it is natural to ask whether the map $t \mapsto \mu_{t}$, where $t$ ranges over a set $\Lambda$ of parameters such that $f_{t}$ has (at least) one SRB measure $\mu_{t}$, is differentiable at 0 . Differentiability should be understood in the sense of Whitney if $\Lambda$ does not contain a neighbourhood of 0 , as suggested by Ruelle [16]. Katok, Knieper, Pollicott, and Weiss 7] gave a positive answer to this differentiability question in the setting of $C^{3}$ families of transitive Anosov flows, showing that $t \mapsto \int \psi d \mu_{t}$ is differentiable, for all smooth $\psi$. If $f_{0}$ is a $C^{3}$ mixing Axiom $\mathrm{A}$ attractor and the family $t \mapsto f_{t}$ is $C^{3}$, Ruelle [15] not only proved that $t \mapsto \int \psi d \mu_{t}$ is differentiable, but also gave an explicit formula, the linear response formula, for the derivative. Of course, in the Anosov and Axiom A cases, $\Lambda$ is a neighbourhood of 0 .

Ruelle [16] suggested that this linear response formula, appropriately interpreted, should hold in much greater generality. Indeed, Dolgopyat [6] obtained the linear response formula for a class of partially hyperbolic diffeomorphisms. In a previous work 3, 4, we found that in the setting of piecewise expanding unimodal interval maps, the SRB measure is differentiable if and only if the path $f_{t}$ is tangent to the

Received by the editors January 22, 2008, and, in revised form, May 27, 2008.

2000 Mathematics Subject Classification. Primary 37C40, 37C30, 37D25, 37E05.

The first author is partially supported by ANR-05-JCJC-0107-01. She wrote part of this paper while visiting the Universidad Católica del Norte, Antofagasta, Chile, whose hospitality is gratefully acknowledged. We thank D. Sands for very helpful comments.

The second author is partially supported by CNPq 470957/2006-9 and 310964/2006-7, FAPESP 2003/03107-9. He thanks the DMA of École Normale Supérieure for hospitality during a visit where a crucial part of this work was done.

(C)2008 American Mathematical Society Reverts to public domain 28 years from publication 
topological class of $f_{0}$, that is, if and only if $\left.\partial_{t} f_{t}\right|_{t=0}$ is horizontal. We emphasize that this setting is not structurally stable. When differentiability holds, Ruelle's candidate for the derivative, as interpreted in [2], gives the linear response formula. (We refer to [2, 3, 4], which also contain conjectures about smooth, not necessarily analytic, Collet-Eckmann maps, for more information and additional references.) Then, Ruelle 17] proved the linear response formula for a class of nonrecurrent analytic unimodal interval maps $f_{t}$, assuming that all $f_{t}$ stay in the topological class of $f_{0}$. (Recall that $f_{t}$ is nonrecurrent if $\inf _{k} d\left(f_{t}^{k}(c), c\right)>0$, where $c$ denotes the critical point.)

In the present work, we consider holomorphic families $f_{t}$ of quadratic-like holomorphic Collet-Eckmann maps. By holomorphic, we mean complex analytic. Our assumptions imply, using classical holomorphic motions, that all $f_{t}$ lie in the same conjugacy class. Generalising one of the arguments in [7, we are able to show that $t \mapsto \int \psi d \mu_{t}$ is real analytic for any real analytic function $\psi$, our main result.

Let us now state our result more precisely. Let $I=[-1,1]$. A $C^{3}$ map $f: I \rightarrow I$ is an $S$-unimodal map if it has $c=0$ as a unique critical point, and $f$ has nonpositive Schwarzian derivative, that is, $\frac{f^{\prime \prime \prime}}{f^{\prime}}-\frac{3}{2}\left(\frac{f^{\prime \prime}}{f^{\prime}}\right)^{2} \leq 0$ except at $c$. An $S$-unimodal map is called Collet-Eckmann if there exist $C>0$ and $\lambda_{c}>1$ so that $\left|\left(f^{n}\right)^{\prime}(f(c))\right| \geq C \lambda_{c}^{n}$ for all $n \geq 1$. In this paper, we shall only consider $S$-unimodal maps with $f^{\prime \prime}(c) \neq 0$.

In Section 2 we shall define precisely the notion of a holomorphic (complex analytic) family of quadratic-like maps in a neighbourhood of $I$ and what all periodic orbits repelling means for such maps, and prove the main result of this work:

Theorem 1.1. Let $t \mapsto f_{t}$ be a holomorphic family of quadratic-like maps in a neighbourhood of $I$, with all periodic orbits repelling. Assume in addition that for each small real $t$ the map $f_{t}$ restricted to $I$ is a (real) Collet-Eckmann S-unimodal map. Then there exists $\epsilon>0$ so that for each real analytic $\psi: I \rightarrow \mathbb{C}$, the map

$$
t \mapsto \int \psi \rho_{t} d x
$$

where $\rho_{t}$ is the invariant density of $f_{t}$, is real analytic on $(-\epsilon, \epsilon)$.

The quadratic-like assumption implies that $f_{t}^{\prime \prime}(c)<0$. The fact that periodic orbits are repelling implies that $f_{t}$ is topologically conjugated with $f_{0}$ : see our use of Mañé-Sad-Sullivan [10] in the beginning of the proof of the theorem in Section 2, Besides Mañé-Sad-Sullivan [10], the other main ingredients of our proof are the results and constructions of Keller and Nowicki [8, which allow us to exploit dynamical zeta functions, following the argument in the work of Katok-KnieperPollicott-Weiss [7, first proof of Theorem 1].

The extension from quadratic-like to polynomial-like is straightforward, and we stick to the nondegenerate case $f^{\prime \prime}(c) \neq 0$ for the sake of simplicity of exposition. As the proof uses only real-analyticity of the holomorphic motions $t \mapsto h_{t}$, it is conceivable that the conclusion of the theorem holds if $f_{t}$ is a real analytic family of quadratic-like maps, using ideas of [1], but this generalisation appears to be nontrivial.

Lyubich's work [9] implies that there are many nontrivial families $f_{t}$ satisfying the assumptions of our theorem. Constructing examples of such families $f_{t}$ is in fact easier, and we sketch the procedure next: Start from two topologically conjugated Collet-Eckmann quadratic-like maps $f$ and $g$ which are not differentiably conjugated. By the result of Przytycki and Rohde [14, they are quasi-conformally 
conjugated. Hence, using a Beltrami path, one can construct a complex analytic family $f_{t}$ of Collet-Eckmann maps containing both $f$ and $g$, with $f_{0}=f$, say. If $f$ and $g$ are real and conjugated in the real line, one can ensure that $f_{t}$ is real for real parameters $t$. If $f$ has negative Schwarzian derivative, then $f_{t}$ has negative Schwarzian derivative for $t$ close to 0 . See also [5] for a specific example and numerical experiments.

\section{Proof of the theorem}

Before we prove the theorem, let us define precisely the objects we are studying:

Definition 2.1. We say that $f_{t}$ is a holomorphic family of quadratic-like maps in a neighbourhood of $I$ if there exists a complex neighbourhood $U$ of $I$ so that $t \mapsto f_{t}$ is a holomorphic map from a complex neighbourhood of zero to the Banach space $B(U)$ of holomorphic functions on $U$ extending continuously to $\bar{U}$, with the supremum norm, such that:

- For real $t$, the map $f_{t}$ is real on $U \cap \mathbb{R}$, with $f_{t}(I) \subset I$ and $f_{t}(-1)=f_{t}(1)=$ -1 .

- There exist simply connected complex domains $W$ and $V$, whose boundaries are analytic Jordan curves, with $I \subset V, \bar{V} \subset U, \bar{V} \subset W$, and so that $f_{0}: V \mapsto W$ is a degree-two ramified covering, with $c=0$ as a unique critical point. That is, $f_{0}: V \mapsto W$ is a quadratic-like restriction of $f_{0}$.

If $f_{t}$ is a holomorphic family of quadratic-like maps in a neighbourhood of $I$, then it is easy to see that for small complex $t$, denoting by $V_{t}$ the connected component of $f_{t}^{-1}(W)$ containing 0 , then $f_{t}: V_{t} \mapsto W$ is a quadratic-like restriction of $f_{t}$ : indeed, $\partial W$ is an analytic Jordan curve, and $f_{0}$ has no critical point on $\partial V$. If $f_{t} \in B(U)$ is close to $f_{0}$, there is a simply connected domain $V_{t}$ close to $V$ such that $f_{t}\left(V_{t}\right)=W$, and the boundary of $\partial V_{t}$ is a Jordan curve, by the implicit function theorem. Then $f_{t}: V_{t} \rightarrow W$ is a quadratic-like extension. We may then give another definition:

Definition 2.2. We say that $f_{t}$ is a holomorphic family of quadratic-like maps in a neighbourhood of $I$ with all periodic orbits repelling if $f_{t}$ is a holomorphic family of quadratic-like maps in a neighbourhood of $I$ so that, for each small complex $t$, the map $f_{t}$ only has repelling periodic orbits in $V_{t}$.

Proof. Since we assumed that all periodic points of $f_{t}$ are repelling, 10, Theorem B] implies that there exists a holomorphic motion of the Julia set $K\left(f_{0}\right)$ of $f_{0}$, that is, a map $h: D \times K\left(f_{0}\right) \rightarrow C$, where $D=\left\{z \in \mathbb{C}|| z \mid<\epsilon_{0}\right\}$ for some $\epsilon_{0}>0$, such that for each $x \in K\left(f_{0}\right)$ the map $t \mapsto h_{t}(x)$ is holomorphic, and for every $t \in D$ the function $x \mapsto h_{t}(x)$ is continuous and injective on $K\left(f_{0}\right)$, with

$$
h_{t} \circ f_{0}=f_{t} \circ h_{t} .
$$

In particular, $h_{t}$ is a homeomorphism from $K\left(f_{0}\right)$ to $K\left(f_{t}\right)$. Note that [10, Theorem B] is quoted for polynomial maps, but the proof immediately extends to polynomial-like. Our assumptions imply that $\left[f_{0}^{2}(0), f_{0}(0)\right]=K\left(f_{0}\right) \cap \mathbb{R}$ and $h_{t}\left(K\left(f_{0}\right) \cap \mathbb{R}\right)=K\left(f_{t}\right) \cap \mathbb{R}=\left[f_{t}^{2}(0), f_{t}(0)\right]$. From now on, we only use real analyticity of $t \mapsto f_{t}(x)$ and $t \mapsto h_{t}(x)$ for $x \in\left[f^{2}(0), f(0)\right]$.

We next claim that our assumptions guarantee that each $f_{t}$ satisfies the technical requirement needed by Keller and Nowicki [8, (1.2)]. Denoting by $\operatorname{var}_{J} \phi$ the total variation of a function $\phi$ on an interval $J$, and writing $f=f_{t}$, we need to check that there is a constant $M>0$ such that: 
a. $M^{-1}<\sup _{I} \frac{|x-c|}{\left|f^{\prime}(x)\right|}+\operatorname{var}_{I} \frac{|x-c|}{\left|f^{\prime}(x)\right|}<M$,
b. $\operatorname{var}_{J_{u}} \frac{|f(x)-f(u)|}{|x-u|\left|f^{\prime}(x)\right|}<M$, where $J_{u}=[-1, u]$ if $u<c$ and $=[u, 1]$ if $u>c$.

Let $\delta_{1}>0$ be such that $\left|f^{\prime \prime}(y)\right|>\left|f^{\prime \prime}(c)\right| / 2$ if $|y-c|<\delta_{1}$. It suffices to prove (a.) and (b.) for $|x-c|<\delta_{1}$ and $|u-c|<\delta_{1}$, and we restrict to such points. Noting that for every such $x \neq c$ there exist $y_{x}, z_{x}$, and $\tilde{z}_{x}$, between $x$ and $c$, so that

$$
\frac{|x-c|}{\left|f^{\prime}(x)\right|}=-\frac{x-c}{f^{\prime}(x)-f^{\prime}(c)}=-\frac{1}{f^{\prime \prime}\left(y_{x}\right)},
$$

and, using $f^{\prime \prime}(x)=f^{\prime \prime}(c)+f^{(3)}\left(z_{x}\right)(x-c)$ and $f^{\prime}(x)=f^{\prime \prime}(c)(x-c)+f^{(3)}\left(\tilde{z}_{x}\right) \frac{(x-c)^{2}}{2}$,

$$
\partial_{x} \frac{|x-c|}{\left|f^{\prime}(x)\right|}=\frac{-f^{\prime}(x)+(x-c) f^{\prime \prime}(x)}{\left(f^{\prime}(x)\right)^{2}}=\frac{(x-c)^{2}}{\left(f^{\prime}(x)\right)^{2}}\left(f^{(3)}\left(z_{x}\right)-\frac{f^{(3)}\left(\tilde{z}_{x}\right)}{2}\right),
$$

the first two conditions hold because $f$ is $C^{3}$. For the third condition, consider $x \geq u>c$ (the other case is symmetric). Since

$$
\frac{f(x)-f(u)}{(x-u) f^{\prime}(x)}=1+\frac{x-u}{f^{\prime}(x)} \frac{f^{\prime \prime}\left(z_{x}\right)}{2}=1+\frac{x-u}{f^{\prime}(x)} \frac{f^{\prime \prime}\left(z_{x}\right)}{2 f^{\prime \prime}\left(y_{x}\right)},
$$

and $0<-\frac{x-u}{f^{\prime}(x)}<-\frac{x-c}{f^{\prime}(x)}$, we get that $\left|\frac{f(x)-f(u)}{(x-u) f^{\prime}(x)}\right|$ is bounded on $[u, 1]$, uniformly in $u$. Finally, since

$$
\partial_{x} \frac{x-u}{f^{\prime}(x)}=\frac{f^{\prime}(x)-(x-u) f^{\prime \prime}(x)}{\left(f^{\prime}(x)\right)^{2}},
$$

analyticity of $f$ implies that $\partial_{x} \frac{x-u}{f^{\prime}(x)}$ changes signs finitely many times, uniformly in $u$, proving (b.).

Also, the results of Nowicki-Sands [13] and Nowicki-Przytycki [12] ensure (see Appendix A that there exist $\lambda_{c}>1, \lambda_{\text {per }}>1, \lambda_{\eta}>1$, and $\epsilon_{1}>0$ such that, for each $|t|<\epsilon_{1}$, there is $C_{t}>0$ with

$$
\left|\left(f_{t}^{n}\right)^{\prime}\left(f_{t}(0)\right)\right| \geq C_{t} \lambda_{c}^{n}, \forall n \geq 1,
$$

and such that for each $x \in I$ with $f_{t}^{p}(x)=x$ for some $p \geq 1$, we have

$$
\left|\left(f_{t}^{p}\right)^{\prime}(x)\right| \geq C_{t} \lambda_{p e r}^{p}
$$

and, finally, setting

$$
\lambda_{\eta}(t):=\liminf _{n \rightarrow \infty}\left\{|\eta|^{-1 / n} \mid \eta \subset I \text { is the largest monotonicity interval of } f_{t}^{n}\right\},
$$

we have

$$
\inf _{|t|<\epsilon_{1}} \lambda_{\eta}(t)>\lambda_{\eta}
$$

In other words, the hyperbolicity constants are uniform in $t$, guaranteeing uniformity when applying the results of Keller and Nowicki [8]. (We choose $\epsilon_{1}<\epsilon_{0}$.)

We now adapt the strategy used in the first proof of [7, Theorem 1]. Fix $\psi$ and, for $x \in I$ such that $f_{0}^{p}(x)=x$ for $p \geq 1$, and for small reals $s$ and $t$, consider

$$
g_{s, t}(x)=\frac{e^{s \psi\left(h_{t}(x)\right)}}{\left|f_{t}^{\prime}\left(h_{t}(x)\right)\right|}
$$

Since $\psi$ is real analytic, the analyticity of $t \mapsto h_{t}$ and of $t \mapsto f_{t}$ together with (2) imply that there is an $\epsilon_{2}>0$ so that, for every periodic point $x \in I$ of period $p \geq 1$ 
for $f$, the function

$$
(t, s) \mapsto g_{s, t}^{(p)}(x):=\frac{e^{s \sum_{k=0}^{p-1} \psi\left(h_{t}\left(f^{k}(x)\right)\right.}}{\left|\left(f_{t}^{p}\right)^{\prime}\left(h_{t}(x)\right)\right|}
$$

is real analytic in $|s|<\epsilon_{2}$ and $|t|<\epsilon_{2}$, uniformly in $x$. We take $\epsilon_{2}<\epsilon_{1}$.

Therefore, the dynamical zeta function defined by

$$
\zeta(s, t, z):=\exp \sum_{p=1}^{\infty} \frac{z^{p}}{p} \sum_{x \in I: f_{0}^{p}(x)=x} g_{s, t}^{(p)}(x)
$$

has the following property: There exists $\delta_{2}>0$ so that for each $|z|<\delta_{2}$ the function $\zeta(s, t, z)$ is real analytic in $|t|<\epsilon_{2},|s|<\epsilon_{2}$, and so that for each $(s, t)$ with $|t|<\epsilon_{2}$, $|s|<\epsilon_{2}$ the map $\zeta(s, t, z)$ is holomorphic and nonvanishing in $|z|<\delta_{2}$.

Now, $h_{t} \circ f_{0}=f_{t} \circ h_{t}$ immediately implies

$$
\zeta(s, t, z)=\exp \sum_{p=1}^{\infty} \frac{z^{p}}{p} \sum_{y \in I: f_{t}^{p}(y)=y} \frac{e^{s \sum_{k=0}^{p-1} \psi\left(f_{t}^{k}(y)\right)}}{\left|\left(f_{t}^{p}\right)^{\prime}(y)\right|} .
$$

Before we proceed, we warn the reader that our parameter $s$ is called $t$ in $[8$, the parameter $\beta$ in 8 ] is $\beta=1$, and our parameter $t$ corresponds to changing the dynamics.

Recall (1, 2, 3) and take $\Theta \in(0,1)$ with

$$
\Theta^{-1}<\min \left\{\lambda_{\eta}, \sqrt{\min \left(\lambda_{c}, \lambda_{p e r}\right)}\right\} .
$$

Keller and Nowicki [8, Theorem 2.1] prove that if $\epsilon_{3} \in\left(0, \epsilon_{2}\right)$ is small enough, then for $|s|<\epsilon_{3}$ and $|t|<\epsilon_{3}$, the transfer operator

$$
\mathcal{L}_{s, t} \varphi(x)=\sum_{\hat{f}_{t}(y)=x} \frac{\omega_{t}(y)}{\omega_{t}(x)} \frac{\exp (s \psi(y))}{\left|\hat{f}_{t}^{\prime}(y)\right|} \varphi(y),
$$

acting on functions of bounded variation on a suitable Hofbauer tower extension $\hat{f}_{t}: \hat{I} \rightarrow \hat{I}$ of $f_{t}$ [8, Section 3], endowed with an appropriate [8, $\left.\S 6.2\right]$ cocycle $\omega_{t}$, is a bounded operator. Note that the cocycle embodies the singularities along the postcritical orbit of $f_{t}$.

If $s=0$, then the spectral radius $\lambda_{0, t}$ of $\mathcal{L}_{s, t}$ is equal to 1 , it is a simple eigenvalue, whose eigenvector gives the invariant density $\rho_{t}$ of $f_{t}$, and the rest of the spectrum is contained in a disc of strictly smaller radius. In addition, the essential spectral radius $\theta_{s, t}$ of $\mathcal{L}_{s, t}$ satisfies $\sup _{|t|<\epsilon_{3},|s|<\epsilon_{3}} \theta_{s, t}<\Theta$, and for each $|t|<\epsilon_{3}$, the spectral radius $\lambda_{s, t}>\Theta$ of $\mathcal{L}_{s, t}$ is an analytic function [8, Prop. 4.2] of $s$.

Note that $\lambda_{s, t}$ is the exponential of the topological pressure of $s \psi-\log \left|f_{t}^{\prime}\right|$ for $f_{t}$ and that $\rho_{t} d x$ is the equilibrium state for $f_{t}$ and $-\log \left|f_{t}^{\prime}\right|$. Now, perturbation theory gives (see [8, (5.2)])

$$
\left.\partial_{s} \log \lambda_{s, t}\right|_{s=0}=\int \psi \rho_{t} d x .
$$

Keller and Nowicki also show [8, Theorem 2.2] that for $|t|<\epsilon_{3}$ and $|s|<\epsilon_{3}$, the power series $\zeta(s, t, z)$ defined by (6) extends meromorphically to the disc of radius $\Theta^{-1}$, and its poles $z_{k}$ in this disc are in bijection with the eigenvalues $\lambda_{k}$ of $\mathcal{L}_{s, t}$, via $\lambda_{k}=z_{k}^{-1}$. In addition, the order of the pole coincides with the algebraic multiplicity of the eigenvalue. By [8, Prop. 4.3 and Lemma 4.5] $\zeta(s, t, z)$ does not 
vanish in the disc of radius $\Theta^{-1}$. It follows that $z \mapsto \zeta(s, t, z)^{-1}$ is holomorphic in the disc of radius $\Theta^{-1}$. This disc contains $\lambda_{s, t}^{-1}$, which is a simple zero.

To end the proof, recalling (77), it suffices to see that $(s, t) \mapsto \lambda_{s, t}$ is real analytic, but this easily follows from Shiffman's [18] real analytic Hartogs' theorem (see Appendix B or [7, Theorem, p. 589]) applied to $d(s, t, z)=\zeta(s, t, z)^{-1}$, which implies that for each $(s, t) \in\left(-\epsilon_{3}, \epsilon_{3}\right) \times\left(-\epsilon_{3}, \epsilon_{3}\right)$ the map $z \mapsto d(s, t, z)$ is holomorphic in $|z|<\Theta^{-1}$. Indeed, by the implicit function theorem, the simple zeroes of $d(s, t, \cdot)$ depend real analytically on $s$ and $t$.

We used the same $\epsilon_{i}$ discs for the $s$ and $t$ variables, but a more careful analysis shows that $\epsilon$ in the statement of the theorem may be selected independently of $\psi$.

\section{APPENDIX A. UnIFORMITY OF THE HYPERBOLICITY CONSTANTS}

Duncan Sands' explanations were instrumental in writing this appendix, and we thank him for that.

We start with a preliminary observation: Let $g$ be an $S$-unimodal ColletEckmann map (with $g^{\prime \prime}(0)<0$, say). Denote by $\lambda_{c}(g), \lambda_{p e r}(g)$, and $\lambda_{\eta}(g)$ the constants defined by (1, 2, 3) (replacing $f_{t}$ by $g$ ). Nowicki and Sands 13] proved that if $g$ is an $S$-unimodal map and $\lambda_{\text {per }}(g)>1$, then $\lambda_{c}(g)>1$. A careful study of their proof shows that $\lambda_{c}(g)>\lambda_{\text {per }}(g)^{\alpha}$, where the exponent $\alpha>0$ only depends on the maximum length $N(g)$ of "almost-parabolic funnels" of $g$ (see [13, Lemma 6.6] for a definition of $N(g)$, which can be bounded by a function of $1 / \log \left(\lambda_{\text {per }}(g)\right)$ and $\left.\sup \left|g^{\prime}\right|\right)$. Since $N(g)$ is in fact invariant under topological conjugacy and $f_{t}$ is topologically conjugated to $f_{0}$, we conclude that $\lambda_{c}\left(f_{t}\right)>\lambda_{\text {per }}\left(f_{t}\right)^{\alpha}$, with $\alpha>0$ uniform in small $t$.

Next, recall that Nowicki and Przytycki 12 proved that if $g$ and $\tilde{g}$ are $S$ unimodal maps, with $g^{\prime \prime}(c) \neq 0$ and $\tilde{g}^{\prime \prime}(c) \neq 0$, say, conjugated by a homeomorphism of the interval and $g$ is Collet-Eckmann, then $\tilde{g}$ is Collet-Eckmann. Take $g=f_{0}$ and $\tilde{g}=f_{t}$. In particular, $f_{t}$ is $C^{2}$ close to $f_{0}$ and $t \mapsto h_{t}$ is smooth. Then it is not very difficult to see that the constants $M=M\left(f_{t}\right)>0, P_{4}=P_{4}\left(f_{t}\right)>0$, and $\delta_{4}=\delta_{4}\left(f_{t}\right)>0$ from the topological characterisation ("finite criticality") of Collet-Eckmann in [12, (4), p. 35]) are uniform in small $t$.

Recall that our assumptions imply $f_{t}^{\prime \prime}(c) \neq 0$ for all small $t$, so that the constant denoted $l_{c}$ in [12] is $l_{c}=2$. Section 2 of [12], and in particular the use of the Koebe principle there, implies that there exists a (universal) function $q: \mathbb{R}_{*}^{+} \times$ $(0,1) \rightarrow(0,1)$ with $q(M, 1 / 4)<1 / 2$ for any $M$ (see [12, Lemma 2.2]) so that

$\lambda_{\text {per }}\left(f_{t}\right)>\left(1-2 q\left(M\left(f_{t}\right), 1 / 4\right)\right)^{-1}$. Therefore, $\lambda_{\text {per }}\left(f_{t}\right)>1$ is uniformly bounded away from 1 for small $t$. The preliminary observation then implies that $\lambda_{c}\left(f_{t}\right)$ is also uniformly bounded in $t$. By [11, Proposition 3.2] (see also [12, p. 35]), this implies a uniform lower bound for $\lambda_{\eta}\left(f_{t}\right)$. Indeed, in the notation of [11, §3], we have $\lambda_{\eta}=\lambda_{5}=\lambda_{4} \geq \lambda_{3}=\lambda_{1} \geq \sqrt{\lambda_{c}}$.

\section{Appendix B. Shiffman's Real analytic Hartogs' extension theorem}

Theorem B.1 ([18). Let $\delta>0$ and $0<r<R$. Assume that

$$
d:(-\delta, \delta)^{2} \times\{z \in \mathbb{C}|| z \mid<R\} \rightarrow \mathbb{C}
$$

satisfies the following conditions:

- for each $(s, t) \in(-\delta, \delta)^{2}$ the map $z \mapsto d(s, t, z)$ is holomorphic in $|z|<R$; 
- for each $|z|<r$ the map $(s, t) \mapsto d(s, t, z)$ is real analytic in $(-\delta, \delta)^{2}$.

Then $d(s, t, z)$ is real analytic on $(-\delta, \delta)^{2} \times\{|z|<R\}$.

Note that the above theorem fails if real analyticity is replaced by $C^{k}$ for $k \leq \infty$.

The theorem holds because $|z|<r$ is not pluripolar in $|z|<R$. Shiffman's result is based on deep work of Siciak [19].

\section{REFERENCES}

1. A. Avila, M. Lyubich, and W. de Melo, Regular or stochastic dynamics in real analytic families of unimodal maps, Invent. Math. 154 (2003) 451-550. MR2018784(2006i:37083)

2. V. Baladi, On the susceptibility function of piecewise expanding interval maps, Comm. Math. Phys. 275 (2007) 839-859. MR2336367 (2008g:37034)

3. V. Baladi and D. Smania, Linear response formula for piecewise expanding unimodal maps, Nonlinearity 21 (2008) 677-711.

4. V. Baladi and D. Smania, Smooth deformations of piecewise expanding unimodal maps, arXiv:0710.1845, preprint, 2007, to appear in Discrete Contin. Dynam. Systems Series A.

5. J. Barnes and J. Hawkins, Families of ergodic and exact one-dimensional maps, Dynam. Systems 22 (2007) 203-217. MR2327993

6. D. Dolgopyat, On differentiability of SRB states for partially hyperbolic systems, Invent. Math. 155 (2004) 389-449. MR2031432 (2005h:37070)

7. A. Katok, G. Knieper, M. Pollicott, and H. Weiss, Differentiability and analyticity of topological entropy for Anosov and geodesic flows, Invent. Math. 98 (1989) 581-597. MR.1022308 (90i:58150)

8. G. Keller and T. Nowicki, Spectral theory, zeta functions and the distribution of periodic points for Collet-Eckmann maps, Comm. Math. Phys. 149 (1992) 31-69. MR1182410 (93i:58123)

9. M. Lyubich, Feigenbaum-Coullet-Tresser universality and Milnor's hairiness conjecture, Ann. of Math. 149 (1999) 319-420. MR.1689333 (2000d:37051)

10. R. Mañé, P. Sad, and D. Sullivan, On the dynamics of rational maps, Ann. Sci. École Norm. Sup. 16 (1983) 193-217. MR732343 (85j:58089)

11. T. Nowicki, Some dynamical properties of S-unimodal maps, Fund. Math. 142 (1993) 45-57. MR:1207470(94c:58111)

12. T. Nowicki and F. Przytycki, Topological invariance of the Collet-Eckmann property for Sunimodal maps, Fund. Math. 155 (1998) 33-43. MR1487986 (99a:58058)

13. T. Nowicki and D. Sands, Nonuniform hyperbolicity and universal bounds for S-unimodal maps, Invent. Math. 132 (1998) 633-680. MR.1625708 (99c:58122)

14. F. Przytycki and S. Rohde, Rigidity of holomorphic Collet-Eckmann repellers, Ark. Mat. 37 (1999) 357-371. MR1714763 (2000i:37064)

15. D. Ruelle, Differentiation of SRB states, Comm. Math. Phys. 187 (1997) 227-241, Differentiation of SRB states: Corrections and complements, Comm. Math. Phys. 234 (2003) 185-190. MR.1463827 (98k:58144), MR 1963142(2004b:37038)

16. D. Ruelle, Application of hyperbolic dynamics to physics: Some problems and conjectures, Bull. Amer. Math. Soc. 41 (2004) 275-278. MR.2058287 (2005b:37038)

17. D. Ruelle, Structure and $f$-dependence of the a.c.i.m. for a unimodal map $f$ of Misiurewicz type, arXiv.org, 2007.

18. B. Shiffman, Separate analyticity and Hartogs theorems, Indiana Univ. Math. J. 38 (1989) 943-957. MR1029683 (91a:32018)

19. J. Siciak, Separately analytic functions and envelopes of holomorphy of some lower dimensional subsets of $C^{n}$, Ann. Polon. Math. 22 (1969) 145-171. MR0252675 (40:5893)

Département de Mathématiques et Applications, UMR 8553, École Normale Supérieure, 75005 Paris, France

E-mail address: viviane.baladi@ens.fr

Departamento de Matemática, icmC-Usp, Caixa Postal 668, São Carlos-SP, CeP 13560-970 SÃO CARLOS-SP, BRAZIL

E-mail address: smania@icmc.usp.br 